\title{
Comparison of thermally and chemically reduced graphene oxides by thermal analysis and Raman spectroscopy
}

\author{
Shereen Farah ${ }^{1} \cdot$ Attila Farkas $^{2} \cdot$ János Madarász $^{3} \cdot$ Krisztina László $^{1}$ (D)
}

Received: 12 October 2019 / Accepted: 16 April 2020 / Published online: 13 May 2020

(c) The Author(s) 2020

\begin{abstract}
Graphene oxide (GO) is not only an intermediate in top-down graphene preparation, but it is also acknowledged for its convenient application when the hydrophobicity of graphene is a drawback. Its preparation through the wet exfoliation of graphite inevitably leads to a product lacking several of the outstanding features of graphene. The destroyed graphene structure can be at least partially reconstructed by thermal and/or wet chemical reduction. Thermally reduced graphene oxide (TRGO) was obtained by mild heat treatment $\left(300{ }^{\circ} \mathrm{C}\right)$ in argon, while chemically reduced graphene oxide (CRGO) was prepared by using a green reagent, ascorbic acid (AA). The parent GO and the two GO derivatives were compared by thermogravimetric analysis coupled with mass spectroscopy and Raman spectroscopy. Both reductive treatments resulted in enhanced thermal stability and higher $\mathrm{C} / \mathrm{O}$ ratio. Although the chemical reduction with AA was more efficient in the elimination of the $\mathrm{O}$ functionalities, it also resulted in significant fragmentation of the graphene-like platelets.
\end{abstract}

Keywords Reduced graphene oxide · Improved Hummers' method · Thermal analysis (TG-DTG, TG-MS) · Raman spectroscopy

\section{Introduction}

Since the first isolation of graphene by Geim and Novoselov et al. [1], the flat two-dimensional (2D) monolayer of carbon atoms has attracted great interest for its excellent electrical, mechanical, optical, and thermal properties [2, 3]. Graphene

Krisztina László

klaszlo@mail.bme.hu

Shereen Farah

shereen.farah@mail.bme.hu

Attila Farkas

farkas.attila@mail.bme.hu

János Madarász

madarasz@mail.bme.hu

1 Department of Physical Chemistry and Material Science, Budapest University of Technology and Economics, Budapest 1521, Hungary

2 Department of Organic Chemistry and Technology, Budapest University of Technology and Economics, Budapest 1521, Hungary

3 Department of Inorganic and Analytical Chemistry, Budapest University of Technology and Economics, Budapest 1521, Hungary has outstanding potential in various environmentally related areas including catalysis, energy storage, and sensor-related applications [4]. Although graphene has proven its ability to be applied in many sectors, its hydrophobicity is often a critical factor in manufacturing. Hydrophilic graphene derivatives such as graphene oxide (GO) and reduced graphene oxides (RGOs) may help to overcome these problems. GO consists of a low number of graphene sheets with structural defects (single vacancies, Stone-Wales defects, etc.) [5, 6] and is decorated with various oxygen-containing functional groups in the basal planes and peripheries [7-10].

GO is most often prepared by oxidative exfoliation of graphite in highly aggressive media [11-15]. The properties of the obtained GO depend on the graphite precursor and the exfoliation method applied and the efficiency of the purification [14, 16-18]. Recently, the intermediates being formed in the improved Hummers exfoliation method were followed by various methods [19]. The active sites of GO can be easily functionalized opening an avenue, e.g., for site-specific applications [7, 20, 21].

Graphene-like properties can be partially recovered by the reduction of GO. Reduction can be performed either by thermal treatment in inert or reductive atmosphere or by wet chemical ways. The chemistry and the properties of the RGO 
depend on the reduction path, i.e., the temperature, duration, reducing agent, concentration, etc.

It was found that thermal reduction of GO may occasionally leads to explosion. The kinetics and mechanism of the thermal reduction of GO are not fully understood, therefore particularly larger samples should be heat treated with special care [22]. When heating GO in argon flow at $800{ }^{\circ} \mathrm{C}$, the oxygen-containing functional groups (typically epoxy, carboxyl and carbonyl) were removed and concurrently small molecules (carbon dioxide, carbon monoxide, water vapor) were released [23]. Sofer et al. investigated the thermal reduction of GO in inert nitrogen and reactive hydrogen atmosphere, respectively, also at $800{ }^{\circ} \mathrm{C}$. Their gas chromatographic analysis coupled with mass spectrometry (GC-MS) revealed that beside the simple molecules listed above various aromatic compounds were also released. Their chemistry and amount were different in the two media and led to RGO products with significantly different $\mathrm{C} / \mathrm{O}$ ratio: as expected, hydrogen gave the higher $\mathrm{C} / \mathrm{O}$ ratio [24]. After thermal reduction in non-reactive Ar flow, the partially stacked GO colloids are restored, but the wrinkled graphene sheets form a loosely stacked graphene-like structure, but the perfect graphene is not re-established even at $2000{ }^{\circ} \mathrm{C}$ [25].

A wide variety of reducing agents can be used in the wet method, such as hydrogen sulfide [26], hydrazine [27, 28], hydroquinone [29], sodium borohydride [30], hydrohalic acids [31], ascorbic acid (AA) [32], or lithium aluminum hydride (LAH) [33]. All these reducing agents have advantages and drawbacks. Although hydrazine is toxic and explosive, it has high efficiency and produces graphene materials with good qualities [27]. A comparative study on the reduction efficiency of GO by AA, hydrazine and sodium borohydride showed that the FTIR peaks representing epoxy and hydroxyl groups decrease after reduction. This phenomenon was more pronounced when GO was treated with AA or hydrazine, the former giving the highest $\mathrm{C} / \mathrm{O}$ ratio from XPS analysis [32]. Sodium borohydride was found efficient in the reduction of carbonyl, epoxy, and hydroxyl groups but only partially reduced carboxyl groups [30]. One of the advantages of AA is that it is nontoxic in contrast to hydrazine and is more stable in water than sodium borohydride. It is one of the most environmentally friendly reducing agent, generally applied in highly basic medium [32].

A comparative study of LAH, sodium borohydride, and hydrazine showed that the three agents reduced GO with different degree. Based on XPS analysis LAH resulted in the highest $\mathrm{C} / \mathrm{O}$ ratio (12), higher than sodium borohydride (9.5). Reduction with hydrazine was also highly efficient $(C / O=11.5)$ and led to the concomitant introduction of nitrogen atom. LAH was also the most effective in the reduction of carboxyl groups compared to hydrazine and sodium borohydride [33].
The binding energy of the oxygen functionalities also depends on their location. The removal of the epoxy and hydroxyl groups from the graphitic sheet is easier than from the defective sites or edges. For their efficient removal, chemical and thermal treatments can be combined [34].

In spite of the different mechanisms of the various reducing agents, no exclusive selectivity can be achieved. Nevertheless, the advantage of both the thermal and wet chemical treatments is that the extent of the reduction can be tuned by the selected agent and the reaction parameters. Thus, RGO with different hydrophilic/hydrophobic properties can be obtained.

For this study GO was prepared by the improved Hummers exfoliation method [14]. RGOs were obtained from this GO by thermal and wet routes. Thermally reduced graphene oxide (TRGO) was obtained by heating freeze-dried GO rods at moderate temperature $\left(300^{\circ} \mathrm{C}\right)$ in argon flow. A green reducing agent, $\mathrm{AA}$ at high $\mathrm{pH}$, was used yielding chemically reduced graphene oxide (CRGO) which was also investigated in freeze-dried state. The behavior of the precursor and the two reduced graphene oxides obtained along these pathways was studied by thermogravimetric analysis coupled with mass spectroscopy (TG/DTA-MS) both in inert and oxidative atmosphere. The occasional reconstruction of the graphitic structure was followed by Raman spectroscopy through the ratio of the $\mathrm{G}$ and $\mathrm{D}$ bands.

\section{Experimental}

\section{Materials and methods}

\section{Sample preparation}

Natural graphite powder (GR) was purchased from Tyn, China, with average particle size of $63 \mu \mathrm{m}$ (95\%). Graphene oxide (GO) was prepared according to the improved Hummers' method [14]. The product was washed several times till neutral $\mathrm{pH}$ was achieved with a yield of $33 \%$. The brown $1 \mathrm{w} / \mathrm{w} \%$ aqueous suspension was kept in an amber bottle. Part of the aqueous GO suspension was freeze-dried $\left(-100{ }^{\circ} \mathrm{C}, 48 \mathrm{~h}\right)$ to obtain GOlyo rods. Thermally reduced graphene oxide (TRGO) was obtained from dry GOlyo after $3 \mathrm{~h}$ at $300{ }^{\circ} \mathrm{C}$ in argon flow $\left(4 \mathrm{dm}^{3} \mathrm{~h}^{-1}\right)$ with a yield of $53 \%$. Chemical reduction of the suspended GO was performed by treating the suspension in $0.02 \mathrm{M}$ aqueous ascorbic acid at $\mathrm{pH} 10\left(95^{\circ} \mathrm{C}, 1 \mathrm{~h}\right)$ [32]. The $\mathrm{pH}$ was set with $\mathrm{cc} \mathrm{NH}_{4} \mathrm{OH}$. The black suspension was washed several times till neutral $\mathrm{pH}$ was achieved. After freeze-drying $\left(-100{ }^{\circ} \mathrm{C}, 72 \mathrm{~h}\right)$, the chemically reduced graphene oxide (CRGO) was obtained with a yield of $63 \%$. The sample preparation is summarized in Fig. 1. 
Fig. 1 Preparation of GO and its derivatives

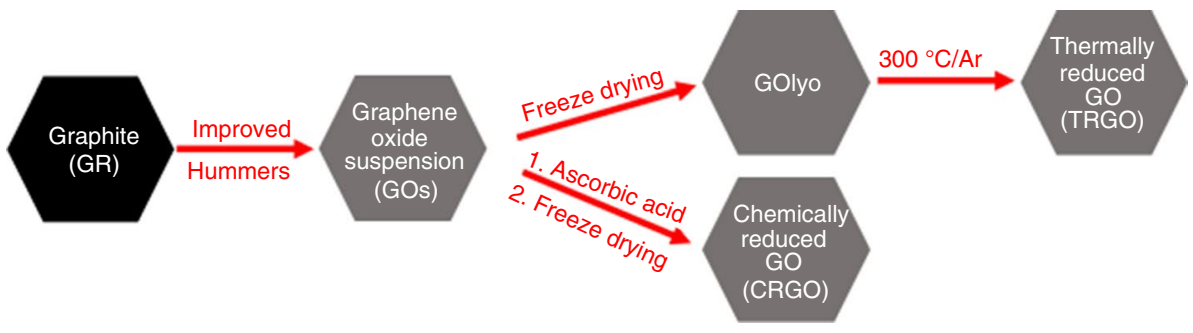

\section{Methods}

The thermal behavior of graphene oxide and its derivatives was accomplished by TG/DTA (SDT 2960, TA Instruments Inc., USA) instrument. TG measurements were conducted under dynamic conditions both in air and nitrogen (flow rate $130 \mathrm{~cm}^{3} \mathrm{~min}^{-1}$ ) with a ramp rate $5^{\circ} \mathrm{C} \min ^{-1}$ up to $110^{\circ} \mathrm{C}$ then $10^{\circ} \mathrm{C} \min ^{-1}$ to $900{ }^{\circ} \mathrm{C}$ (except GR, which was heated up to $950{ }^{\circ} \mathrm{C}$ and GOlyo up to $650^{\circ} \mathrm{C}$ in air). Approximately, $4 \mathrm{mg}$ of each sample was placed in an open Pt crucible. Parallel with the TG measurement, the analysis of the evolved volatile products was carried out on Thermostar GSD 200T (Balzers Instruments) quadruple mass spectrometer (MS) equipped with a Chaneltron detector and coupled to the TG/ DTA instrument. The gas transfer lines to the spectrometer were kept at $200{ }^{\circ} \mathrm{C}$. Data collection was carried out with QuadStar 422v7.02 software in scanning mode in the range of $m / z=1-300$, and in multiple ion detection mode (MID) on 64 selected $\mathrm{m} / \mathrm{z}$ channels.

Raman spectra were recorded with a LabRAM (Horiba Jobin-Yvon) instrument having a $\lambda=532 \mathrm{~nm} \mathrm{Nd-YAG} \mathrm{laser}$ source (laser power at the focus point is $15 \mathrm{~mW}$ ). Filter of $0.6 \mathrm{OD}$ was used to reduce excitation beam energy reducing the chance of sample degradation. The exposure time was 120 s per point. Results shown here are the averages of five different locations in the samples. A $1800 \mathrm{~g} \mathrm{~mm}^{-1}$ grating dispersed the Raman-scattered light before it reached the CCD detector. LabSpec 5 software was applied for parameter optimization and data collection.

\section{Results and discussion}

The thermal stability of the pristine GR, GOlyo, and the two reduced derivatives was studied up to $900{ }^{\circ} \mathrm{C}$ in inert and oxidative media (except GR, which was heated up to $950{ }^{\circ} \mathrm{C}$ and GOlyo up to $650^{\circ} \mathrm{C}$ in oxidative media), using nitrogen and air flows, respectively. Figure 2 compares the TG curves and their first derivatives.

GR, as expected, was thermally stable and had only a $2.3 \%$ mass reduction until $900{ }^{\circ} \mathrm{C}$ in $\mathrm{N}_{2}$ atmosphere (Fig. 2 GR). Mass spectrometry revealed that this loss was due to release of sulfur dioxide (Fig. 3 GR). That is, the natural graphite used contains traces of sulfur or its derivatives.
The TG/DTG curves in air show that the oxidation of the graphite starts already around $700{ }^{\circ} \mathrm{C}$ and by $950{ }^{\circ} \mathrm{C}$ the loss is about $70 \%$. The volatile products were identified as carbon dioxide $(\mathrm{m} / \mathrm{z}=12,22,44,45$ and 46) and sulfur dioxide $(m / z=46,64)$. Although all the listed fragments were experimentally detected, for simplicity, only the $m / z=44$ and 64 were plotted for $\mathrm{CO}_{2}$ and $\mathrm{SO}_{2}$, respectively. No burning was observed and $\mathrm{CO}$ evolution if any was below the detection limit.

GOlyo is significantly less stable due to the high concentration of surface oxygen groups. The $\mathrm{C} / \mathrm{O}$ atomic ratio from XPS measurement was 2.6 [36]. The $5 \%$ loss at $100{ }^{\circ} \mathrm{C}$ in both gases implies the release of adsorbed water retained on the hydrophilic surface (Fig. 2 GOlyo). The run of the two TG curves is overlapping up to $200{ }^{\circ} \mathrm{C}$ where a significant mass loss occurs in both media. In nitrogen, this step is followed by a slow continuous decay with a slope of ca $0.03 \%{ }^{\circ} \mathrm{C}^{-1}$ reaching a total mass loss of $63 \%$ by $900{ }^{\circ} \mathrm{C}$. On the other hand, in air a second, steep step occurs in the $400-600{ }^{\circ} \mathrm{C}$ range leading to the complete oxidation of the sample. Mass spectrometry revealed the evolution of carbon dioxide $(\mathrm{m} / \mathrm{z}=12,22,44,45$ and 46$)$ and sulfur dioxide $\left(m / z=48,49,50,64,65\right.$ and 66) (Fig. 3 GOlyo). The $\mathrm{CO}_{2}$ released around $200{ }^{\circ} \mathrm{C}$ may come from carboxylic groups [35], while the second set of the $\mathrm{CO}_{2}$ related signals detected only in air escorts the complete oxidation of the carbon. The lack of $\mathrm{SO}_{2}$ above $800{ }^{\circ} \mathrm{C}$ indicates that the sulfur content of the natural graphite precursor was eliminated during the wet exfoliation. At the same time, the use of sulfuric acid in the Hummers process led to the entrapment of sulfur species which were detected at much lower temperature, between 200 and $300{ }^{\circ} \mathrm{C}$.

As mentioned in Introduction part, the properties of the reduced $\mathrm{GO}$ depend on the reduction path. The mild thermal route increased the $\mathrm{C} / \mathrm{O}$ atomic ratio to 5.7 , while the $\mathrm{AA}$ treatment was more effective resulting in a $C / O$ ratio of 7.3 [36]. Indeed, comparison of Fig. 2 TRGO, CRGO exposes that the thermal behavior of the two reduced GOs are not identical.

Accordingly, TRGO in $\mathrm{N}_{2}$ shows a limited but gradual mass loss as high as $33 \%$ by $900{ }^{\circ} \mathrm{C}$ releasing traces of $\mathrm{CO}_{2}$ and, in the two different temperature ranges, also $\mathrm{SO}_{2}(m / z=48$ and 64). In air, the sharp mass loss is due to evolving carbon dioxide $(m / z=12,22,44,45$ and 46) in the 

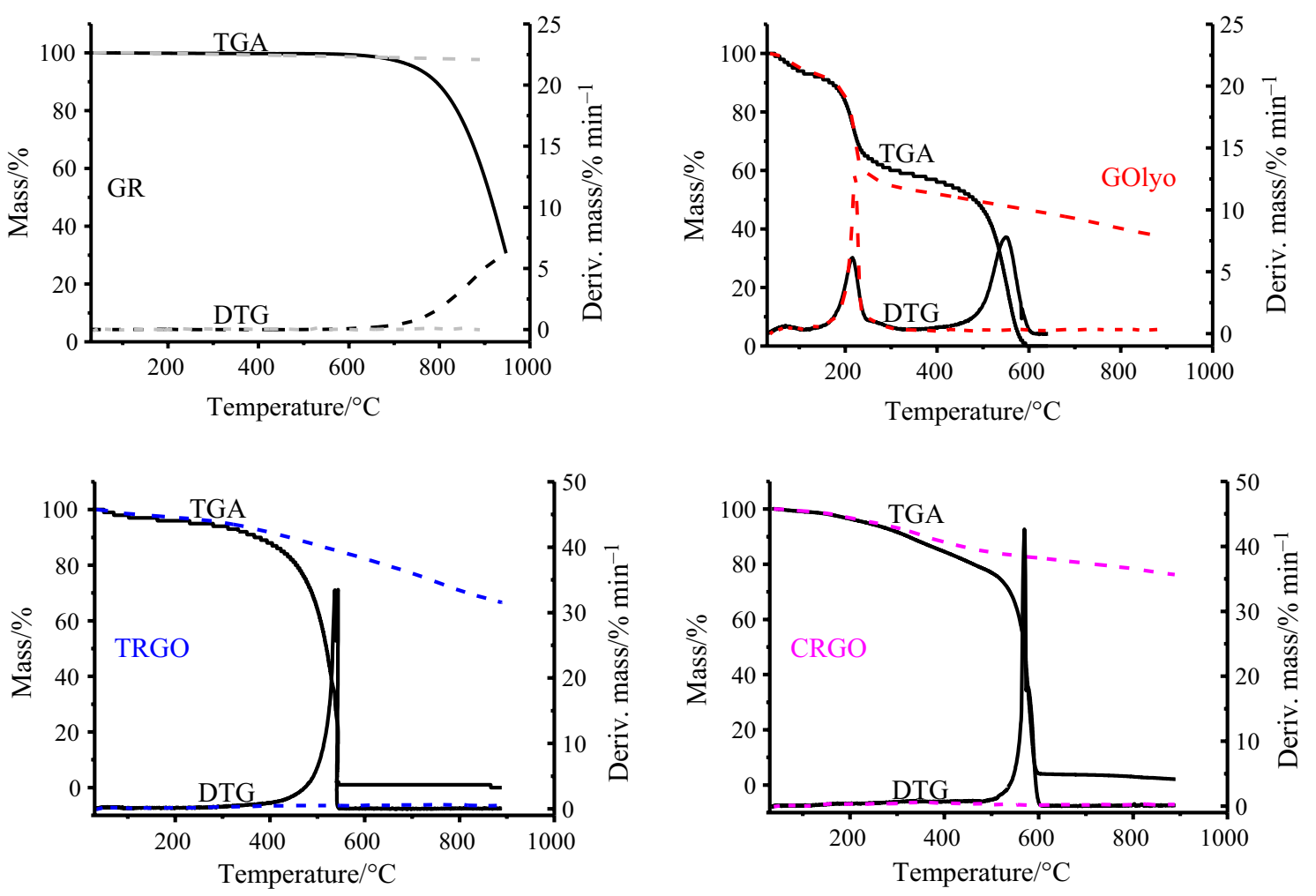

Fig. 2 TG/DTG curves of GR, GOlyo, TRGO, and CRGO in air (solid line) and in $\mathrm{N}_{2}$ gas (dashed line) flow, respectively

$450-550{ }^{\circ} \mathrm{C}$ range beside sulfur dioxide $(\mathrm{m} / \mathrm{z}=64)$ between 300 and $450{ }^{\circ} \mathrm{C}$ and above $800{ }^{\circ} \mathrm{C}$ (Figs. 2 TRGO, 3 TRGO).

In $\mathrm{N}_{2}$ gas flow, the mass of the more reduced CRGO decreased continuously to a total mass loss of $24 \%$ while releasing only sulfur dioxide $(\mathrm{m} / \mathrm{z}=48,49,50,64,65$ and 66) (Figs. $2 \mathrm{CRGO}, 3 \mathrm{CRGO}$ ). In air, the slow $\mathrm{SO}_{2}$ evolution was below the detection limit, only carbon dioxide was observed $(\mathrm{m} / \mathrm{z}=12,22,44,45$ and 46$)$ in the temperature range of $300-620^{\circ} \mathrm{C}$. It is worthwhile to mention that while both GOlyo and TRGO were completely oxidized in air by the end of the measurement, CRGO retained $2 \%$ of its original mass when heated to $900{ }^{\circ} \mathrm{C}$.

Raman spectroscopy is a versatile and therefore widely used tool to identify and characterize the chemical and physical properties of carbon materials, as due to the conjugation of the carbon-carbon double bonds, they give high Raman intensities [37, 38]. Two principle features of the spectrum, the $\mathrm{G}$ and $\mathrm{D}$ bands, are used most often to follow the changes during various treatments. The band appearing around $1587 \mathrm{~cm}^{-1}$ is the $\mathrm{G}$ band. It corresponds to the inplane vibrational mode that involves $s p^{2}$ hybridized carbon atoms that comprises the graphene sheet. The disorder or the defect band, i.e., the D band is generally weak in graphite and high-quality graphene. It is related to a ring breathing mode from $s p^{2}$ carbon rings near a graphene edge or a defect.
The amount of defects present in a sample is generally quantified by the intensity ratio of these bands. The structural changes occurring in our samples during the various treatments were also followed by Raman spectroscopy. Figure 4 shows the $800-1800 \mathrm{~cm}^{-1}$ range of the recorded spectra. As expected, the $\mathrm{G}$ bands appear around $1587 \mathrm{~cm}^{-1}$, and the position of the $\mathrm{D}$ bands is in the $1347-1356 \mathrm{~cm}^{-1}$ range. The spectrum of the pristine graphite displays a strong $\mathrm{G}$ and a weak $\mathrm{D}$ band with an intensity ratio $I_{\mathrm{G}} / I_{\mathrm{D}} 6.0 \pm 0.5$.

The Hummers' exfoliation, including also insertion of oxygen functional groups, significantly destroys the graphitic order as reflected not only by the results discussed above but also by the significantly diminished intensity ratio to $1.18 \pm 0.01$. The reduction processes further suppress the value of $I_{\mathrm{G}} / \mathrm{I}_{\mathrm{D}}$. For TRGO, this ratio $1.08 \pm 0.01$ is close to that for GOlyo implying that the moderate thermal treatment probably does not enter new defects. A comparison of Raman spectra of reduced GO obtained after thermal treatment in hydrogen and nitrogen atmospheres showed that the inert atmosphere resulted in less defect than the reactive one [24]. Much significant decrease in the $I_{\mathrm{G}} / I_{\mathrm{D}}$ value $(0.75 \pm 0.03)$ was found in CRGO. Such phenomenon is commonly found after chemical GO reduction, and is attributed to the fragmentation of the $s p^{2}$ domains upon reduction of the $\mathrm{GO}$ [27]. 
Fig. 3 Mass spectra of the detected volatile products (only fragments $m / z, 44$ and 64 for $\mathrm{CO}_{2}$ and $\mathrm{SO}_{2}$, respectively, are plotted). GR, GOlyo, TRGO, and CRGO in air (solid line) and $\mathrm{N}_{2}$ gas (dashed line) flow, respectively. The curves are shifted along the ion current axis for better visibility
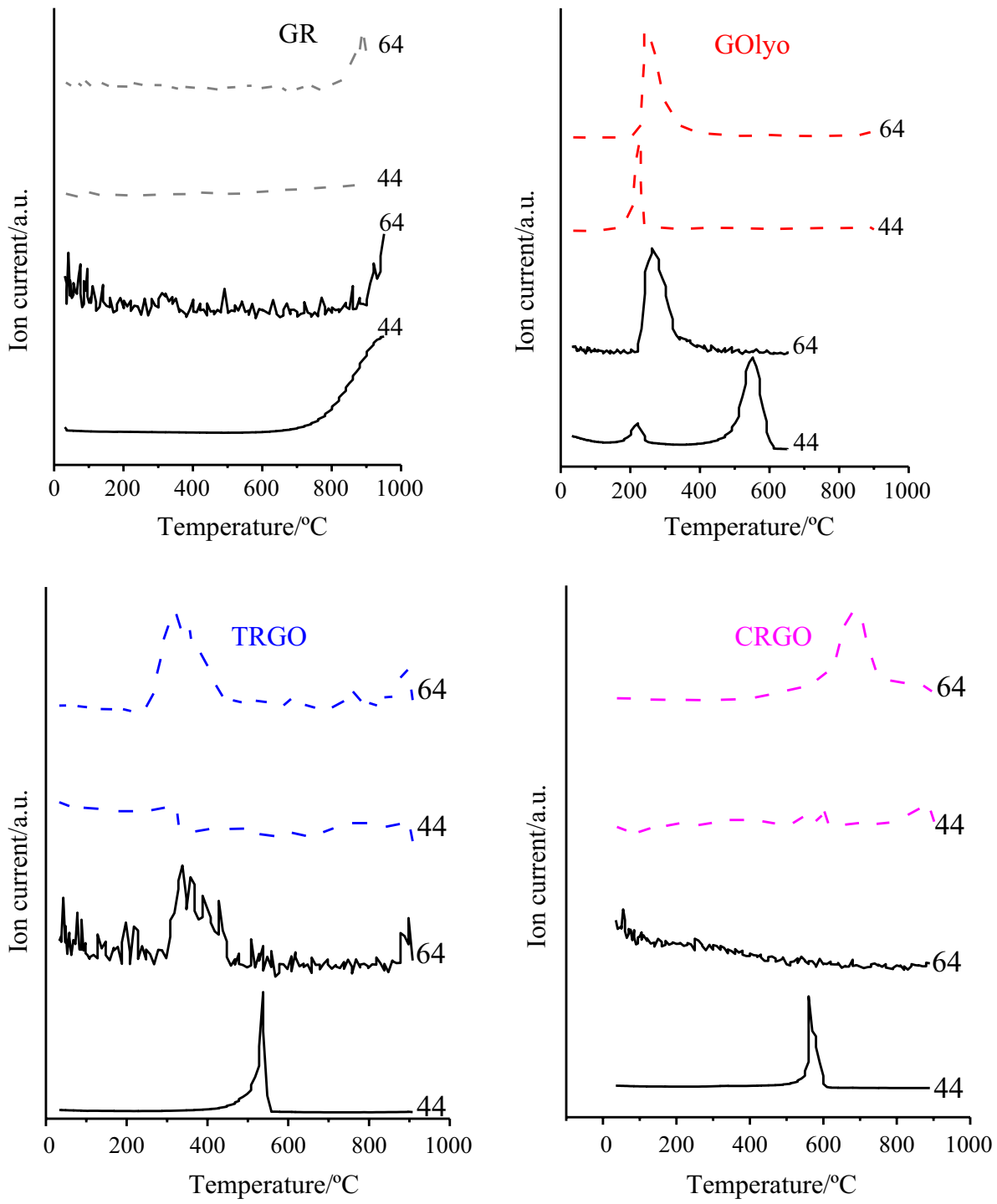

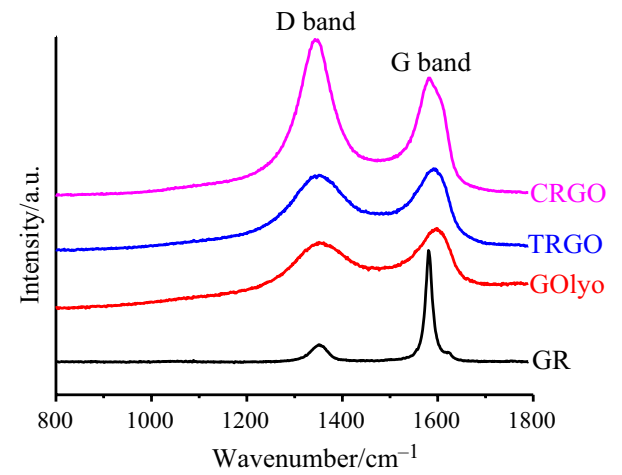

Fig. 4 Raman spectra of graphite (GR), freeze-dried graphene oxide (GOlyo) and the thermally and chemically reduced graphene oxides (TRGO and CRGO, respectively)

\section{Conclusions}

The chemical exfoliation performed according to the improved Hummers' method results in severe damage in the ideal graphene sheets. The oxygen functionalities and the defects could be at least partially eliminated by physical and/or chemical reduction. Comparison of the products from a $300{ }^{\circ} \mathrm{C}$ thermal treatment in inert argon atmosphere and from chemical reduction by ascorbic acid (AA) in basic medium was performed by thermal methods assisted by mass spectrometry and Raman spectroscopy. Although the $\mathrm{ClO}$ ratio improves during both treatments, the reduced derivatives are different according to their chemical and thermal properties. The trend in the $I_{\mathrm{G}} / I_{\mathrm{D}}$ intensity ratios also corroborates that the two reduction methods compared in this study led to products different in chemistry, thermal behavior, and imperfections. The 
chemical reductive treatment caused further imperfections by the simultaneous fragmentation of the platelets.

Acknowledgements We extend our warm thanks to Ms. Andrea Domán for her advices and to Mr. G. Bosznai for invaluable technical assistance. This work was supported by the NRDI Fund K128410 and by the BME-Nanotechnology FIKP Grant of EMMI (BME FIKPNAT). The work is related to the Nanoporous and Nanostructured Materials for Medical Applications (H2020-MSCA-RISE-2016-734641 NanoMed), and VEKOP-2.3.2-16-2017-00013 Projects. The latter is jointly supported by the EU and by Hungary, co-financed by the European Regional Development Fund. SF is grateful to the Stipendium Hungaricum scholarship program.

Funding Open access funding provided by Budapest University of Technology and Economics (BME).

Open Access This article is licensed under a Creative Commons Attribution 4.0 International License, which permits use, sharing, adaptation, distribution and reproduction in any medium or format, as long as you give appropriate credit to the original author(s) and the source, provide a link to the Creative Commons licence, and indicate if changes were made. The images or other third party material in this article are included in the article's Creative Commons licence, unless indicated otherwise in a credit line to the material. If material is not included in the article's Creative Commons licence and your intended use is not permitted by statutory regulation or exceeds the permitted use, you will need to obtain permission directly from the copyright holder. To view a copy of this licence, visit http://creativecommons.org/licenses/by/4.0/.

\section{References}

1. Novoselov KS, Geim AK, Morozov SV, Jiang D, Zhang Y, Dubonos SV, Grigorieva IV, Firsov AA. Electric field in atomically thin carbon films. Science. 2004;306:666-9.

2. Rao CNR, Sood AK, Subrahmanyam KS, Govindaraj A. Graphene: the new two-dimensional nanomaterial. Angew Chem Int Ed. 2009;48:7752-77.

3. Rao CNR, Sood AK, Voggu R, Subrahmanyam KS. Some novel attributes of graphene. J Phys Chem Lett. 2010;1:572-80.

4. Zhaoa X, Jiaqiang E, Wuc G, Denga Y, Han D, Zhanga B, Zhang Z. A review of studies using graphenes in energy conversion, energy storage and heat transfer development. Energy Convers Manag. 2019;184:581-99.

5. Radovic LR, Silva-Tapia AB, Vallejos-Burgos F. Oxygen migration on the graphene surface. 1. Origin of epoxide groups. Carbon. 2011;49:4218-25.

6. Murugan L, Lakshmipathi S, Bhatia SK. Influence of in-plane StoneThrower-Wales defects and edge functionalisation on the adsorption of $\mathrm{CO}_{2}$ and $\mathrm{H}_{2} \mathrm{O}$ on graphene. RSC Adv. 2014;4:39576-87.

7. Dreyer DR, Park S, Bielawski CW, Ruoff RS. The chemistry of graphene oxide. Chem Soc Rev. 2010;39:228-40.

8. Pacilé D, Meyer JC, Rodríguez AF, Papagno M, Gomez-Navarro C, Sundaram RS, Burghard M, Kern K, Carbone C, Kaiser U. Electronic properties and atomic structure of graphene oxide membranes. Carbon. 2011;49:966-72.

9. He H, Klinowski J, Forster M, Lerf A. A new structural model for graphite oxide. Chem Phys Lett. 1998;287:53-6.

10. Szabó T, Berkesi O, Forgó P, Josepovits K, Sanakis Y, Petridis D, Dékány I. Evolution of surface functional groups in a series of progressively oxidized graphite oxides. Chem Mater. 2006;18:2740-9.

11. Brodie BC. On the atomic weight of graphite. Philos Trans R Soc Lond. 1859;149:249-59.
12. Staudenmaier L. Verfahren zur Darstellung der Graphitsäure. Ber Dtsch Chem Ges. 1898;31:1481-7.

13. Hummers WS Jr, Offeman RE. Preparation of graphitic oxide. J Am Chem Soc. 1958;80:1339.

14. Marcano DC, Kosynkin DV, Berlin JM, Sinitskii A, Sun Z, Slesarev A, Alemany LB, Lu W, Tour JM. Improved synthesis of graphene oxide. ACS Nano. 2010;4:4806-14.

15. Eigler S, Grimm S, Hof F, Hirsch A. graphene oxide: a stable carbon framework for functionalization. J Mater Chem A. 2013;1:11559-62.

16. Botas C, Álvarez P, Blanco C, Santamaria R, Granda M, Ares P, Rodríguez-Reinoso F, Menéndez R. The effect of the parent graphite on the structure of graphene oxide. Carbon. 2012;50:275-82.

17. Shojaeenezhad SS, Farbod M, Kazeminezhad I. Effects of initial graphite particle size and shape on oxidation time in graphene oxide prepared by Hummers' method. J Sci Adv Mater Dev. 2017;2:470-5.

18. Chua CK, Sofer Z, Pumera M. Graphite oxides: effects of permanganate and chlorate oxidants on the oxygen composition. Chem Eur J. 2012;18:13453-9.

19. Justh N, Berke B, László K, Szilágyi IM. Thermal analysis of the improved Hummers' synthesis of graphene oxide. J Therm Anal Calorim. 2018;131:2267-72.

20. Chen D, Feng H, Li J. Graphene oxide: preparation, functionalization, and electrochemical applications. Chem Rev. 2012;112:6027-53.

21. Chua CK, Pumera M. Chemical reduction of graphene oxide. A synthetic chemistry viewpoint. Chem Soc Rev. 2014;43:291-312.

22. Qiu Y, Guo F, Hurt R, Kulaots I. Explosive thermal reduction of graphene oxide-based materials: mechanism and safety implications. Carbon. 2014;72:215-23.

23. Chen CM, Huang JQ, Zhang Q, Gonga WZ, Yang QH, Wanga MZ, Yanga YG. Annealing a graphene oxide film to produce a free standing high conductive graphene film. Carbon. 2012;50:659-67.

24. Sofer Z, Jankovský O, Simek P, Sedmidubsk D, Šturala J, Kosina J, Miksova R, Macková A, Mikulics M, Pumera M. Insight into the mechanism of the thermal reduction of graphite oxide: deuteriumlabeled graphite oxide is the key. ACS Nano. 2015;9:5478-85.

25. Wang S, Ábrahám D, Vallejos-Burgos F, László K, Geissler E, Takeuchi K, Endo M, Kaneko K. Distorted graphene sheet structurederived latent nanoporosity. Langmuir. 2016;32:5617-22.

26. Hofmann U, Frenzel A. The reduction of graphite oxide by hydrogen sulphide. Kolloid-Zeitschript. 1934;68:149-51.

27. Stankovich S, Dikin DA, Piner RD, Kohlhaas KA, Kleinhammes A, Jia Y, Wu Y, Nguyen ST, Ruoff RS. Synthesis of graphene-based nanosheets via chemical reduction of exfoliated graphite oxide. Carbon. 2007:45:1558-65.

28. Park S, An J, Potts JR, Velamakanni A, Murali S, Ruoff RS. Hydrazine-reduction of graphite- and graphene oxide. Carbon. 2011;49:3019-23.

29. Wang G, Yang J, Park J, Gou X, Wang B, Liu H, Yao J. Facile synthesis and characterization of graphene nanosheets. J Phys Chem C. 2008;112:8192-5.

30. Shin HJ, Kim KK, Benayad A, Yoon SM, Park HK, Jung IS, Jin MH, Jeong HK, Kim JM, Choi JY, Lee YH. Efficient reduction of graphite oxide by sodium borohydride and its effect on electrical conductance. Adv Funct Mater. 2009;19:1987-92.

31. Pei S, Zhao J, Du J, Ren W, Cheng HM. Direct reduction of graphene oxide films into highly conductive and flexible graphene films by hydrohalic acids. Carbon. 2010;48:4466-74.

32. Fernánde-Merino MJ, Guardia L, Paredes JI, Villar-Rodil S, SolísFernández P, Martínez-Alonso A, Tascón JMD. Vitamin C is an ideal substitute for hydrazine in the reduction of graphene oxide suspensions. J Phys Chem C. 2010;114:6426-32.

33. Ambrosi A, Chua CK, Bonanni A, Pumera M. Lithium aluminum hydride as reducing agent for chemically reduced graphene oxides. Chem Mater. 2012;24:2292-8. 
34. Gao X, Jang J, Nagase S. Hydrazine and thermal reduction of graphene oxide: reaction mechanisms, product structures, and reaction design. J Phys Chem C. 2009;114:832-42.

35. Zielke U, Hüttinger KJ, Hoffman WP. Surface-oxidized carbon fibers: I. Surface structure and chemistry. Carbon. 1996;34:983-98.

36. Berke B. Thermosensitive hydrogel - carbon nanoparticle composites. PhD Theses. Budapest University of Technology and Economics, Budapest, Hungary. 2017.

37. Kudin KN, Ozbas B, Schniepp HC, Prud'Homme RK, Aksay IA, Car R. Raman spectra of graphite oxide and functionalized graphene sheets. Nano Lett. 2008;8:36-41.
38. Wu JB, Lin ML, Cong X, Liu HN, Tan PH. Raman spectroscopy of graphene-based materials and its applications in related devices. Chem Soc Rev. 2018;47:1822-73.

Publisher's Note Springer Nature remains neutral with regard to jurisdictional claims in published maps and institutional affiliations. 